\title{
Video cascade accumulation of the total solar eclipse on Svalbard 2015
}

\author{
Fred Sigernes $^{1,8}$, Pål Gunnar Ellingsen ${ }^{1,8}$, Noora Partamies ${ }^{1,8}$, Mikko Syrjäsuo ${ }^{1,8}$, Pål Brekke ${ }^{2,8}$, \\ Silje Eriksen Holmen ${ }^{1,8}$, Arne Danielsen ${ }^{3}$, Bernt Olsen ${ }^{4}$, Xiangcai Chen ${ }^{1,8}$, Margit Dyrland ${ }^{1}$, Lisa Baddeley ${ }^{1,8}$, \\ Dag Arne Lorentzen ${ }^{1,8}$, Marcus Aleksander Krogtoft ${ }^{5}$, Torstein Dragland ${ }^{5}$, Hans Mortensson ${ }^{5}$, Lisbeth Smistad ${ }^{5}$, \\ Craig J. Heinselman ${ }^{6}$, and Shadia Habbal ${ }^{7}$ \\ ${ }^{1}$ The University Centre in Svalbard (UNIS), 9171 Longyearbyen, Norway \\ ${ }^{2}$ Norwegian Space Centre, Oslo, Norway \\ ${ }^{3}$ Brages 2, 1540 Vestby, Akershus, Norway \\ ${ }^{4}$ Norwegian Broadcasting Corporation (NRK) Troms and Finnmark, Troms $\emptyset$, Norway \\ ${ }^{5}$ Lufttransport AS, Longyearbyen, Norway \\ ${ }^{6}$ EISCAT Scientific Association, Kiruna, Sweden \\ ${ }^{7}$ Institute for Astronomy, University of Hawaii, Honolulu, USA \\ ${ }^{8}$ The Birkeland Centre for Space Science (BCSS), University of Bergen, Bergen, Norway
}

Correspondence to: Fred Sigernes (freds@unis.no)

Received: 17 June 2016 - Published in Geosci. Instrum. Method. Data Syst. Discuss.: 1 September 2016

Revised: 22 December 2016 - Accepted: 28 December 2016 - Published: 13 January 2017

\begin{abstract}
This work presents a novel image accumulation filter technique that reveals small-scale features and details from intense luminosity or high dynamic range (HDR) video recordings. It was discovered and developed from the analyses of the Norwegian Broadcasting Corporation (NRK) film of the total solar eclipse that occurred Friday 20 March 2015 in Longyearbyen $\left(78^{\circ} \mathrm{N}, 15^{\circ} \mathrm{E}\right)$ on Svalbard, Norway. The result of the filter is fused with a HDR image of the corona and the Solar Dynamic Observatory (SDO) image of the solar disk.
\end{abstract}

\section{Introduction}

Stacking or accumulating camera frames is a well-known technique in astrophysics (see Berry and Burnell, 2005). The track-and-stack technique is an effective method to obtain long exposures from many short ones of faint deep-sky objects while tracking. Accumulation will reduce noise and increase the dynamical range. An inexpensive web camera sensor is capable of capturing a large number of faint and noisy exposures that can be stacked into sharp and clear images of deep-sky objects. Free software such as RegiStax (2008) is widely used to align, stack, and process astronomical images. Another benefit of high frame rate and short exposures is that it can be used to minimize atmospheric effects such as seeing (Law et al., 2006; Baldwin et al., 2008).

In this study we present a modified accumulation technique where the target is not only faint but also intense. The intensities of a total eclipse extend from faint background sky conditions in the outer corona to several orders of magnitude brighter intensities close to the solar limb. In order to image the full spatial extent of the event it was necessary to make a sequence of variable exposures with two camera systems. Our filter focuses on the bright chromosphere and the inner corona using data from a professional video camera. In our approach, each individual video frame is processed prior to accumulation to produce a final high dynamic range (HDR) image. In addition, a custom-assembled telescope using a digital single-lens reflex (DSLR) camera head is used to capture and stack images to produce a HDR image of the outer corona. 


\section{Target: the total solar eclipse on Svalbard 2015}

The total solar eclipse on Friday 20 March 2015 started in the western Atlantic, $650 \mathrm{~km}$ west of Canada's Labrador coast and $450 \mathrm{~km}$ south of the southern tip of Greenland. It then raced across the Atlantic Ocean touching land at only two places: the Faroe Islands (between Scotland and Iceland) and the Svalbard Archipelago.

In Longyearbyen $\left(78^{\circ} \mathrm{N}, 15^{\circ} \mathrm{E}\right)$, Svalbard, the first contact, the start of the partial eclipse, started at 09:11:53 UT. About 59 min later, at 10:10:43 UT the second contact took place, marking the start of totality. After a mere $2 \mathrm{~min}$ and $27 \mathrm{~s}$, the third contact occurred at 10:13:10 UT, which marked the end of totality and the disappearance of the corona. This was followed by a partial phase of about another $59 \mathrm{~min}$ before fourth contact and the end of the solar eclipse at 11:12:21 UT.

\section{Experimental setup}

Two cameras were used to capture the totality. A professional digital video camera captured 448 frames while a coronal telescope connected to a DSLR camera head captured $20 \mathrm{im}$ ages during the totality.

\subsection{The digital video camera}

The event was filmed by the Norwegian Broadcasting Corporation (NRK) at Nordlysstasjonen, the old auroral station in Adventdalen. The station is located $\sim 4 \mathrm{~km}$ east of Longyearbyen. The film was broadcasted in real time on the internet with close to 600000 followers.

The NRK Sony camera model PXW-X500 was mounted on a tripod and the Sun was tracked manually. The camera is shown in Fig. 1. The lens was a Canon x 36 super zoom lens with the aperture set to f/5.6. Fully zoomed in, the effective focal length was close to $1000 \mathrm{~mm}$. An Baader AstroSolar safety film was used as a protection filter prior to totality. The exposure time and aperture were manually controlled by the operator.

\subsection{The coronal HDR telescope}

In order to image the full extent of the corona a Nikon D7000 DSLR camera mounted on a $400 \mathrm{~mm}$ focal length telescope was set up at Nordlysstasjonen. Figure 2 shows the experimental setup. The telescope is a triplet-lens apochromatic refractor with an aperture of $80 \mathrm{~mm}$ from the company SkyWatcher, model Esprit-80ED. A field flattener corrector is installed between the triplet and the camera head to match and optimize the illumination of the sensor chip. The telescope was mounted on an azimuth-elevation tracker, from the company iOptron, model Minitower II. The assembled system tracked the total eclipse with a nominal maximum angular error of 0.1 arcsec.

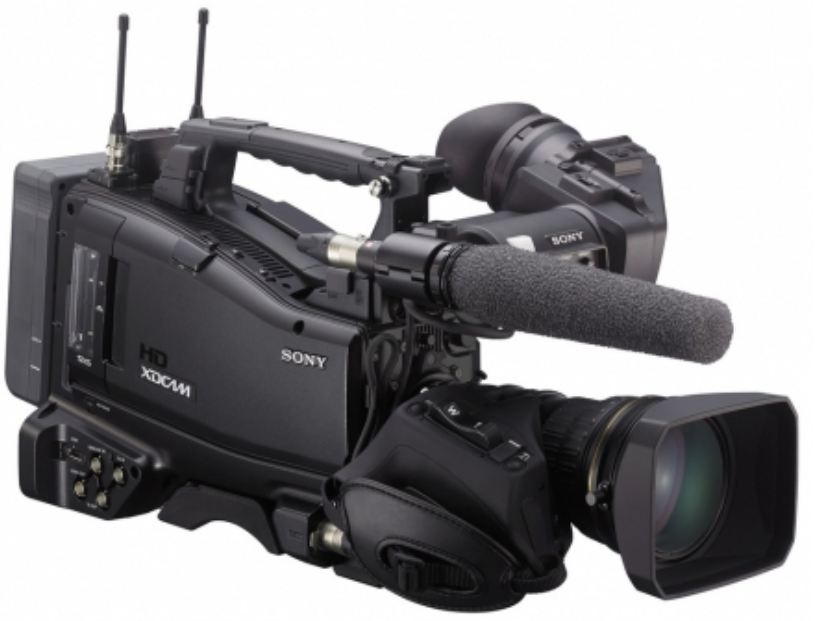

Figure 1. Sony model PXW-X500 professional camcorder. Source: (http://www.sony.com).

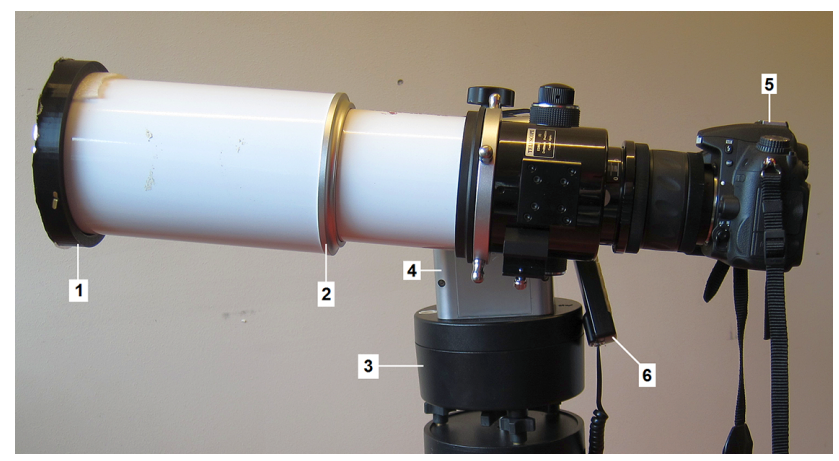

Figure 2. The coronal high dynamic range (HDR) eclipse telescope. (1) Astro-Baader solar protection filter (ND=5), (2) SkyWatcher Esprit 80ED telescope, (3) tripod head, (4) azimuthelevation tracker, (5) Nikon D7000 DSLR camera, and (6) tracker controller.

Twenty images of the totality were taken with variable exposure time ranging from 0.002 up to $2 \mathrm{~s}$ at ISO 100. After alignment of the sequence, images with the same exposure were median filtered to reduce noise. The open-source software Luminance HDR (2015) was used to produce a final HDR image. Tone-mapping was used to improve the visual appearance employing the methods developed by Debevec and Malic (1997) and Mantiuk et al. (2006). The technique is well known in photography as exposure bracketing.

\section{The video filter algorithm}

There are challenges in processing the video camera data. First it is necessary to align each frame in the video to stabilize it. Secondly, a method to sum the frames must be chosen that is independent of exposure and gain variations of the camera. 


\subsection{Alignment of images}

If we define $I_{0}(x, y)$ and $I_{j}(x, y)$ as two frames in a sequence of $j=0$ to $(N-1)$ images, where $(x, y)$ are the pixel coordinates, the spatial shift between the frames can be found using the Fourier shift theorem (Reddy and Chatterji, 1996). The displacement between two images is found by locating the coordinates of the maximum value in the real part of the inverse Fourier transform (FT) of the ratio

$R=F_{0} \cdot \bar{F}_{j} /\left|F_{0} \cdot F_{j}\right|$,

where $F_{0}=F T\left(I_{0}\right)$ and $F_{j}=F T\left(I_{j}\right)$. For local optimization at sub-pixel scale, the maximum shift correlation between $I_{0}$ and $I_{j}$ is found by sub-pixel displacements of 0.1 within a window of \pm 10 pixels in both $x$ and $y$ direction. The linear Pearson correlation coefficient function and the float number shift routine by Lindler (1992) in IDL (Interactive Data Language) are used.

Note that rotation and scale changes due to atmospheric turbulence are neglected in the above calculations. Image $I_{0}$ should be kept fixed as a reference shift frame. Otherwise, the sequence will drift incrementally. In the following we assume that the frames are aligned and denoted $I_{j}(x, y)$ for simplicity.

\subsection{Accumulation of video sequence}

The video frames have small intensity level changes due to both instrument effects and manual adjustments by the camera crew. The target, the total eclipse, is assumed to be stable in intensity during the time the video was captured. Thus, we can use one of the images as the reference and mitigate the instrument effects by taking advantage of the time series. A simple linear model to estimate intensity changes between each color channel is defined as

$I_{0}(x, y)=\alpha_{j} \cdot I_{j}(x, y)+\beta_{j}$.

Here $\alpha_{j}$ and $\beta_{j}$ are defined as the effective software gain and background level, respectively. If we choose pixel values where the target is well defined in shape and stable in intensity, such as the diagonal crossing the center of the Sun, the above coefficients can be estimated for each frame by the least absolute deviation (LAD) method. The IDL function LADFIT.PRO by Press et al. (1992) is used for this purpose. The method is robust and fast. In this study, we have assumed a linear response from the camera allowing a very trivial correction of fluctuations in gain and background levels by using Eq. (2). If the true intensity response curve is known, the intensities should first be converted into a linear scale to minimize estimation errors. Table 1 shows that the calculated values of $\alpha_{j}$ and $\beta_{j}$ are indeed fairly stable with low standard deviations for $N=448$ video frames of the totality. This corresponds to a recording time of $17.92 \mathrm{~s}$ of the totality where the camera was not moved by the operator. The

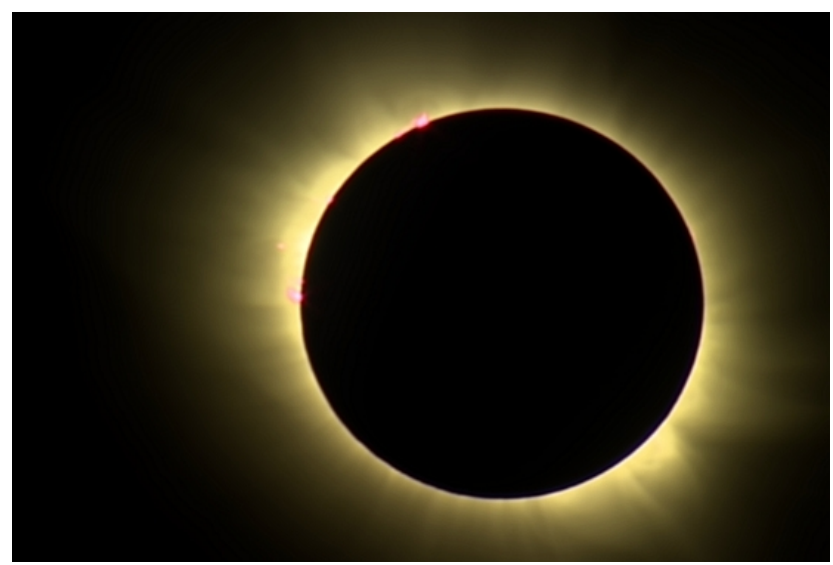

Figure 3. Accumulated color video frames of the total eclipse on Svalbard, 20 March 2015. A total of 448 frames of the NRK video are used.

camera was fixed with the eclipse moving in the field of view only due to the rotation of Earth.

Next, the accumulated frames are now defined as

$I(x, y)=\sum_{j=0}^{N-1} \alpha_{j} \cdot I_{j}(x, y)+\beta_{j}=\sum_{j=0}^{N-1} I_{j}^{s}(x, y)$.

If $j=0$, then $\alpha_{0}=1$ and $\beta_{0}=0$. The reference intensity $I_{0}$ is then chosen to be the first frame in the sequence, although it can also be any other frame.

Figure 3 shows the result of applying Eqs. (1)-(3). Note the intense pink-colored bright features that rise out of the background continuum, localized close to the solar limb. These emissions are from hydrogen and helium and are associated with prominences. Coronal streamers are also clearly detected, but structures close to the limb appear too blurry to be identified.

\subsection{High-pass cascading}

Equation (3) may be modified to include a filter that enhances small-scale edges in each of the frames in the sequence. A high-frequency emphasis filter, $F$, is known to enhance small-scale features and edges can be detected by the use of a histogram-scale Laplacian filter, $S$. The next step is to add the filtered result to the original frame

$I_{j}^{c}=I_{j}^{S}+S\left(F\left(I_{j}^{S}\right)\right)=I_{j}^{S}+S\left(k \cdot I_{j}^{S}-L\left(I_{j}^{S}\right)\right)$,

where $L$ is a low-pass filter and $k=1.1$ the amplification factor recommended by Gonzales and Woods (1992). Higher values of $k$ tend to increase the background too much. In IDL the low-pass filter $L$ is the SMOOTH.PRO function. It uses the boxcar average of a specified pixel width, $w$. SHARPEN.PRO by Fanning (2003) is the Laplacian filter used. The IDL code for Eq. (4) then becomes

$I_{j}^{c}=I_{j}^{s}+\operatorname{SHARPEN}\left(1.1 \cdot I_{j}^{s}-\operatorname{SMOOTH}\left(I_{j}^{s}, w\right)\right)$. 
Table 1. Calculated average color channel software gain $(\alpha)$ and background values $(\beta)$ according to Eq. (2) for $N=448$ frames of the NRK video sequence. $\sigma_{\alpha}$ and $\sigma_{\beta}$ are the corresponding standard deviation for $\alpha$ and $\beta$, respectively.

\begin{tabular}{lrrrr}
\hline Color channel & Average $\alpha$ & Standard deviation $\sigma_{\alpha}$ & Average $\beta$ & Standard deviation $\sigma_{\beta}$ \\
\hline Red & 1.006 & 0.007 & -25.58 & 0.48 \\
Green & 1.004 & 0.007 & -23.27 & 0.27 \\
Blue & 1.020 & 0.016 & -20.02 & 0.27 \\
\hline
\end{tabular}

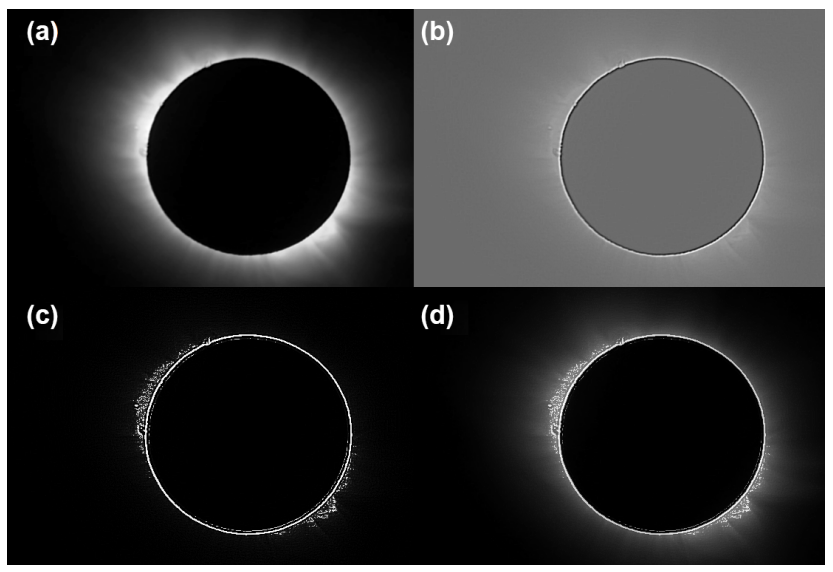

Figure 4. High-pass cascade emphasis filter applied to single green channel NRK video frame. (a) is input video frame, (b) highfrequency emphasis-filtered frame, (c) edge enhancement of (b), and (d) is (a) and (c) added.

The net accumulated result of Eq. (3) combined with Eq. (4) is named a high-pass cascade emphasis filter:

$I^{\prime}(x, y)=\sum_{j=0}^{N-1} I_{j}^{c}(x, y)$.

Figure 4 shows an example of how the above filter works on a single green channel frame for $w=5$. The high-frequency emphasis filter increases noise and sharp intensity transitions in the image. The latter is enhanced by the gradient filter. The net result is added to the original frame. The detected transition points appear in Fig. $4 \mathrm{c}$ to be randomly aligned with the small-scale features that are seen in Fig. 4b. The variation must be caused by scintillation or any other highfrequency change in camera response and noise. Accumulation of a large number of frames should solve this problem.

\section{Results and discussion}

The accumulated high-pass cascade emphasis filter images are visualized in Fig. 5 as a function of boxcar width, $w$. It is clear that the accumulation of filtered frames makes the intense blurry chromospheric regions close to the solar limb appear more structured. Chromospheric loops, spicules, plumes, and prominences are now identified. These features

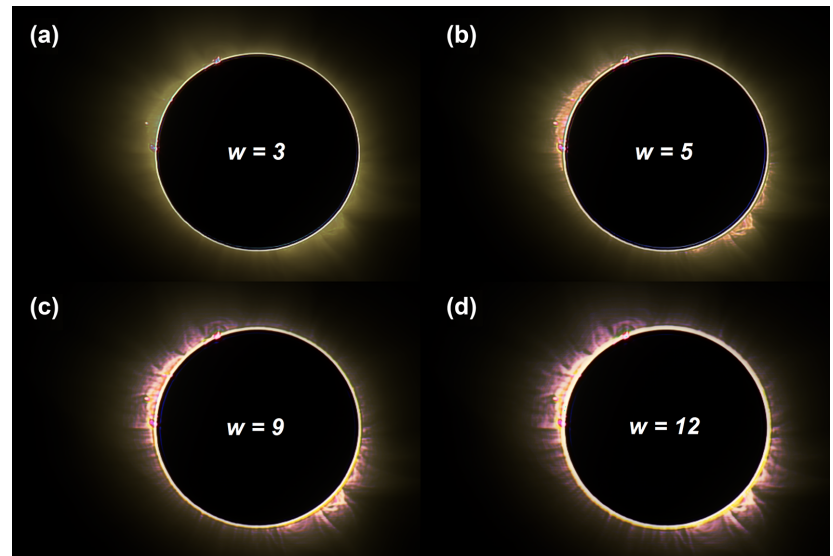

Figure 5. Accumulated high-pass cascade-filtered color images. Boxcar filter sizes in pixels are for panels (a) $3 \times 3$, (b) $5 \times 5$, (c) $9 \times 9$, and (d) $12 \times 12$. A total of 448 color frames of the NRK video are used. The apparent color changes are predominantly caused by processing the frames without a rigorous camera calibration, which is required to measure absolute colors.

have edges and abrupt changes in intensity representing the high-frequency components of the image. The choice of smoothing mask or boxcar size in the low-pass filter determines the level of detail detected. The effect is seen as an increase of width of the circular solar limb as the pixel width $w$ increases. The best results to identify loops and spots are obtained with $w \in[3,5]$. Higher values tend to emphasize larger-scale edges further out in the corona, with less detail in the chromosphere. It should be noted that our technique could be improved by applying a Fourier transform-based low-pass filter to reduce sharp intensity edge effects. There is also a color shift from faint green-yellow to pink with increasing boxcar width in Fig. 5 associated with change in color balance in the composite images, indicating that our technique is not conservative.

The use of wavelets has become a popular method to enhance details in images (Berry and Burnell, 2005). By interactively recombining wavelet transforms at different spatial scales and layers, small-scale features are enhanced and large-scale shading effects are reduced. In order to compare with our cascade filter, the wavelet module of the program RegiStax (2008) is applied to the stacked frame of the aligned video sequence. Both the cascade and the wavelet filter re- 


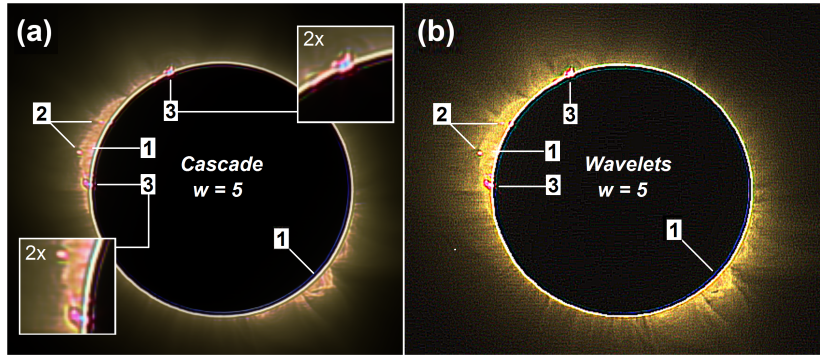

Figure 6. Two filter techniques side by side. (a) Accumulated highpass cascade-filtered color image. A total of 448 color frames of the NRK video are used with a boxcar width of $w=5$. Identified features are (1) loops and spicules region, (2) bright plumes, and (3) prominences. Two zoom windows show the prominences magnified two times. (b) Wavelets enhanced image using the RegiStax (2008) software.

sults are shown side by side in Fig. 6 for a boxcar width of $w=5$. The spatial enhanced image is a combination of two layers of Gaussian wavelets. It is clear, even though the image appears to be noisy, that the same features are detected with the wavelet technique as with the cascade filter. The identified loops, spicules, plumes, and prominences are all known features that appear in the chromosphere. Also note the difference in the colors. Each color channel is independently processed in the cascade filter before the creation of the composite RGB. The emphasized edges and features have spatially distinct color difference as a function of color channel. The prominences appear to be spatially multi-colored with a clear distinct blue core close to the solar limb surrounded by a red plume with yellow or weak green outward borders. The loops and spicules are more orange to red in appearance. The blue and red colors are most likely associated with hydrogen Balmer line emissions $\left(H_{\gamma}, H_{\beta}\right.$ and $\left.H_{\alpha}\right)$. The yellow to green color is a mixture of the $H_{\alpha}$ and the $\mathrm{He}\left(D_{3}\right)$ lines. These are the strongest line emissions from prominences in the visible spectrum (see Slocum, 1912).

It must be emphasized that the wavelet transform is a powerful tool since it can produce an unlimited range of spatial frequencies and scales, but it requires interactive user input to produce the final image. Our attempt to reproduce the event might not be the optimum choice of wavelet scheme. The cascade filter does not require any interactive feedback, except for choice of boxcar size. On the other hand, a fused interactive combination of cascading as a function of boxcar size could also be used to emphasize features and structures with different size and scale. The novelty in our simple technique is the accumulated effect of adding edge detection to the high-frequency emphasis component of each individual frame in a sequence of images. Only basic low- and high-pass filters are used, which makes the filter easy to implement in any high-level program language such as IDL or MATLAB.

Finally, it is now possible to compose an image of the Sun using the video accumulation, the HDR image from the tele-

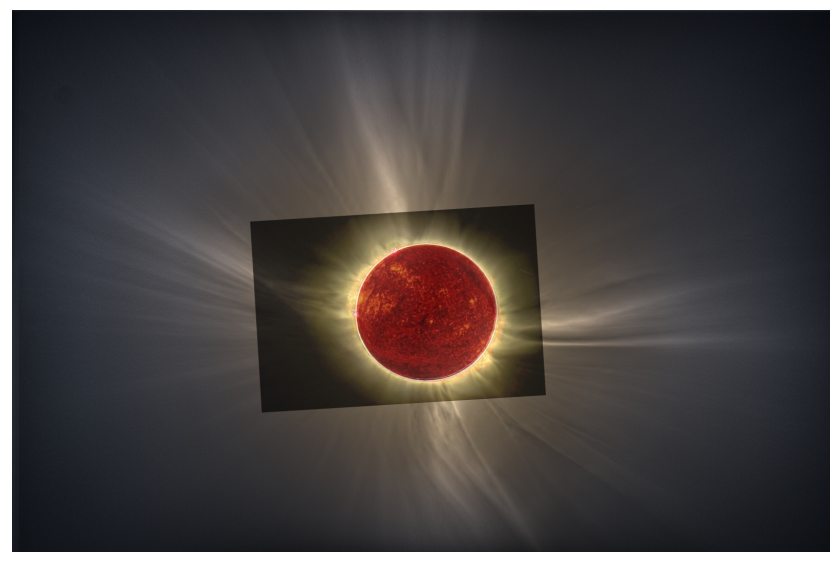

Figure 7. Composite image of the total eclipse as seen from Longyearbyen, 20 March 2015. The white light-colored corona is composed of 20 snapshots from a Nikon D7000 at ISO 100 with exposure times in the range $0.002-2 \mathrm{~s}$. The inner yellow image with dark background is a video accumulation of 448 frames by NRK applying a high-frequency emphasis cascade filter. The solar disk is filled with a red-colored Helium II image at $304 \mathrm{~nm}$ from the Solar Dynamics Observatory (SDO).

scope, and the Helium II Solar Dynamic Observatory (SDO) image at $304 \mathrm{~nm}$. The last was obtained at 10:24:20 UT, which is only $670 \mathrm{~s}$ after the end of the totality in Longyearbyen. The result is shown in Fig. 7. The HDR image is fused with the accumulated image in Fig. 3 and the filter image in Fig. 5a. The SDO image and the video accumulations are resized and rotated to match the HDR image. The program Paint.net for Microsoft Windows was used in the fusion. The matching criterion is based on aligning the two prominences located in the upper left quadrant and the Helmet streamers identified in both the video accumulation and in the coronal HDR image. The SDO image is rotated $23^{\circ}$ clockwise and rescaled to match the diameter of the solar disk. As a result, the intense chromospheric regions in the SDO image align up with the detected loop region in the video accumulation.

Note that if we knew the power of the cascade filter prior to the eclipse, then the video sequence should have been recorded by the DSLR camera of the coronal telescope instead of the NRK video camera. The video frames will then match and align with the HDR image. No rotation between the different camera systems would then be necessary.

The final composed image is an astonishing snapshot of the corona and the outer chromosphere. Four large Helmet streamer belts are seen that extended almost out of our field of view, close to five solar radii. The belts are also structured with curved or twisted appearance, especially the upwardand downward-directed ones. The high number of Helmet streamers and their tortuous complex shapes are closely related to the solar activity cycle (Low, 1996). The solar activity was in other words high during the total eclipse on Svalbard in 2015 . 


\section{Conclusions}

The principal results obtained in the study can be summarized as follows.

1. The total solar eclipse event on Svalbard on 20 March 2015 gave us a unique opportunity to image the upper parts of the Sun's atmosphere. High dynamic range imaging revealed four large structured coronal helmet streamer belts that fold beyond our field of view, close to five solar radii.

2. A novel high-pass cascade emphasis filter technique is presented that is capable of distinguishing multi-colored features such as loops, spicules, plumes, and prominences from the intense and blurry regions of the NRK video.

3. Based on our result, we encourage future eclipse photographers to include - in addition to standard bracketing - $\mathrm{a} \sim 15 \mathrm{~s}$ high-frame-rate video recording of the event. This makes it possible to apply our filter to enhance the high-luminosity regions of the inner corona and chromosphere.

\section{Data availability}

The authors of the paper state that all the data are publicly available. The raw data for this paper can be downloaded here: http://kho.unis.no/Eclipse/Rawdata/Rawdata.zip.

Competing interests. The authors declare that they have no conflict of interest.

Acknowledgement. We wish to thank the Solar Dynamics Observatory (SDO) for their excellent webpage (http://sdo.gsfc.nasa.gov) and free access to solar images. We also thank Kjellmar Oksavik from the Birkeland Centre for Space Science (BCSS) for fruitful discussions and media support during the eclipse.

Edited by: A. Benedetto

Reviewed by: R. Michell and three anonymous referees

\section{References}

Baldwin, J. E., Warner, P. J., and Mackay, C. D.: The point spread function in Luck Imaging and variations in seeing on short timescales, Astron. Astrophys., 480, 589-597, doi:10.1051/0004-6361:20079214, 2008.

Berry, R. and Burnell, J.: The Handbook of Astronomical Image Processing, Willmann-Bell, Inc., 130-141, ISBN 0-943396-824, 2005.

Debevec, P. E. and Malik, J.: Recovering high dynamic range radiance maps from photographs, SIGGRAPH 97 Conf. Proc., 369378, doi:10.1145/258734.258884, 1997.

Fanning, D.: Coyote's Guide to IDL Programming, http://www. idlcoyote.com, 2003.

Gonzalez, R. C. and Woods, R. E.: Digital Image processing, Addison Wesley World Student series, 196-197, ISBN 0-201-60078$1,1992$.

Law, N. M., Mackay, C. D., and Baldwin, J. E.: Lucky imaging: high angular resolution imaging in the visible from the ground*, Astron. Astrophys., 446, 739-745, doi:10.1051/00046361:20053695, 2006.

Low, B. C.: Solar Activity and the Corona, Sol. Phys., 167, 217 265, doi:10.1007/BF00146338, 1996.

Lindler, D.: The IDL Astronomy User's Library, http://idlastro.gsfc. nasa.gov/, 1992.

Luminance HDR: A complete open source solution for HDR photography, http://qtpfsgui.sourceforge.net/, 2015.

Mantiuk, R., Myszkowski, K., and Seidel, H.-P.: A perceptual framework for contrast processing of high dynamic range images, ACM Transactions on Applied Perception, 3, 286-308, DOI:10.1145/1166087.1166095, 2006.

Press, W. H., Teukolsky, S. A., Vetterling, W. T., and Flannery, B. P.: Numerical recipes in C, The Art of Scientific Computing, Second Edition, Cambridge University Press, 703-705, ISBN 0-52143108-5, 1992.

Reddy, B. S. and Chatterji, B. N.: An FFT-based technique for translation, rotation, and scale-invariant image registration, IEEE T. Image Process., 5, 1266-1271, doi:10.1109/83.506761, 1996.

RegiStax: Free image processing software, http://www.astronomie. be/registax/, 2008.

Slocum, F.: The Study of Solar Prominences, Popular Astronomy, 20, 409-414, 1912. 